\title{
Complications in de Quervain surgery; the importance of multi-disciplinary team approach before and after surgery
}

Keywords: de Quervain, tenosynovitis, surgery, complications, CRPS, MDT, therapy

Abbreviations: CRPS, complex regional pain syndrome; MDT, multi-disciplinary team; VAS, visual analog scale; APL, abductor pollicis longus; EPB, extensor pollicis brevis

\section{Case}

A 41year old female accountant presented to the hand clinic in Salisbury, UK three years after a release of the first dorsal extensor compartment in her left dominant wrist due to de Quervain's tenosynovitis. The initial procedure, done elsewhere, was complicated by bleeding and significant post-operative swelling leading to an acute carpal tunnel syndrome for which the patient declined surgical intervention. Later, a complex regional pain syndrome (CRPS) ${ }^{1,2}$ developed. She was referred three years later with chronic pain in the left wrist extending proximally into her forearm, a poorly functioning hand and inability to extend or move her thumb due to severe pain.

On examination within the Multi-Disciplinary Team (MDT), the hand had features of continuous CRPS with swollen fingers with a shiny appearance, diffuse allodynia, and pain with light touch especially over the scar (8/10 on the visual analog scale (VAS)) associated with dizziness, nausea and visual disturbance. Finkelstein test, which initially is used to diagnose de Quervain's tenosynovitis, was still positive. The scar was tethered to the underlying structures There was a positive Tinel sign over the carpal tunnel, yet a nerve conduction study failed to show any objective compression to the median nerve at the wrist. The range of motion at the wrist was mildly reduced, movement of the thumb was severely reduced and grip strength was markedly reduced, witha grip strength ratio ${ }^{3}$ of $14 \%$ compared to the contralateral hand (measured with a Jamar hand dynamometer) and $25 \%$ for lateral pinch. She used the right hand for all functional tasks and scored 29 and 34 respectively for intrusion and avoidance on the Impact of Event Scale-Revised. ${ }^{4}$

Based on clinical symptoms and signs the following contributing factors were diagnosed: signs of incomplete de Quervain release complicated by scarring of the superficial radial nerve, continuous CRPS, signs of median nerve involvement and significant centralisation issues. She was offered an MDT approach to her situation including a reoperation combined with a planned rehabilitation programme.

At operation tenolysis was done to the abductor pollicis longus (APL) and to the extensor pollicis brevis (EPB), which in turn had not been released in a separate sub-compartment. Branches of the superficial radial nerve encased within the scar were neurolysed over a $10 \mathrm{~cm}$ segment and finally a carpal tunnel release was done where the median nerve was released from tethering to the transverse carpal ligament.

Pre-operatively she was planned within the MDT for intensive post-operative physiotherapy with a six week programme including
Volume 10 Issue 4 - 2018

\author{
Oestreich K,' Nicolaou M, ${ }^{2}$ Wareham P, $^{3}$ \\ Lindau TR ${ }^{4}$ \\ 'Consultant Plastic and Hand Surgeon, The Birmingham \\ Children's Hospital, Birmingham, UK \\ ${ }^{2}$ Consultant Plastic Surgeon, Salisbury District Hospital, \\ Salisbury, UK \\ ${ }^{3}$ Director of Physiotherapy, Wessex Rehabilitation Centre, \\ Salisbury, UK \\ ${ }^{4}$ Professor, Consultant Hand Surgeon, Pulvertaft Hand Centre, \\ Royal Derby Hospital, UK
}

Correspondence: Oestreich K, Consultant Plastic and Hand Surgeon, The Birmingham Children's Hospital, Birmingham, UK, Email kerstin.oestreich@nhs.net

Received: April 24, 2018| Published: July 20, 2018

repeat desensitisation exercises to address her hypersensitivity and sympathetic responses to touch, oedema management, progressive range of motion and strengthening exercises for her thumb and wrist and functional gripping activities for return to normal life.

Furthermore, she was rehabilitated via the "Motor Imagery Programme" of laterality exercises aiming to increase her ability to recognise left and right hands appropriately followed by 'imagined movements' and lastly mirror therapy (Figure 1). ${ }^{5}$ This was aimed to address centralisation problems, ${ }^{6}$ which was further addressed with psychological counselling.

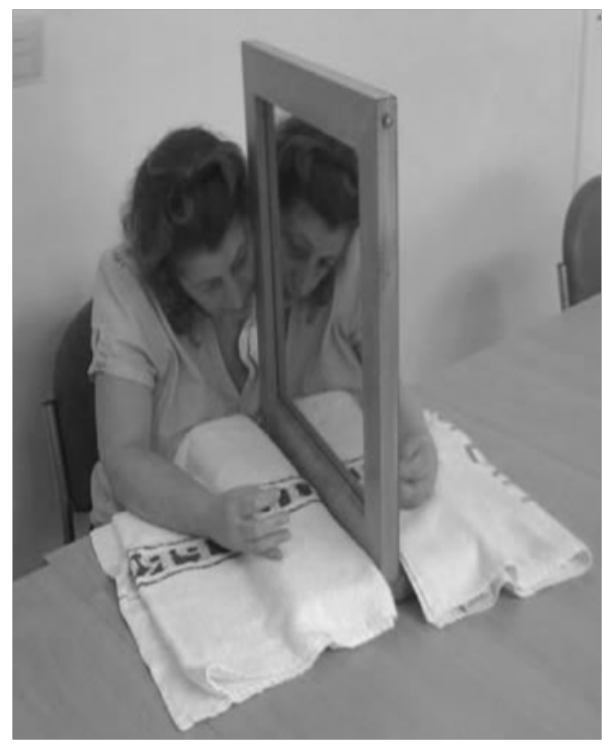

Figure I A patient undergoing mirror therapy. The patient is "observing" the illusion of symmetrical movement of the affected hand (left) by moving of the non-affected hand (right). 
On discharge, her nerve related symptoms had greatly improved, she could recognise left and right hands quickly and accurately, had returned to previous sports activities and reported being more positive in professional and domestic situations. Grip strength ratio improved $^{3}$ to $81 \%$ (preop $14 \%$; Jamar) and $70 \%$ for lateral pinch (preop 25\%)

\section{Conclusion}

Release of de Quervain tenosynovitis is a common procedure, most often operated on by junior hand surgeons. Nevertheless, abnormal anatomy with a separate compartment for $\mathrm{EPB}^{7}$ has to be looked for and sub-compartments to be released. The superficial radial nerve has to be protected and hemostasis has to be done appropriately. This is known to most surgeons, yet complications, such as the one in our case, show that any procedure can go horribly wrong.

If faced with complications such as this one, always approach this professionally with your multi-disciplinary-team (MDT) addressing all aspects of the patient's symptoms and signs. The MDT identifies and score problems pre-operatively and outlines a post-operative plan to treat all aspects of the complication. A simple grip strength ratio, before and after, has been shown to be as good an outcome measure as DASH (Disability Arm Shoulder Hand) and is an easy way of monitoring the recovery after any type of surgery. ${ }^{3}$

If the complication, such as in this case with tethered superficial radial and median nerves, escalates into a full-blown CRPS (complex regional pain syndrome), then neurolysis should be done in addition to all other treatments for CRPS including pain regime for neurogenic pain, intensive rehabilitation, mirror therapy as well as psychological input. ${ }^{2}$ It is important to be aware that prolonged disuse of a limb may also cause reorganization of its representation in the somatosensory cortex ${ }^{8}$ where more advanced therapy may be necessary. In summary, our case was successful due to a pre- and post-operative MDT approach combined with aggressive surgery dealing with all contributing factors causing CRPS.

\section{Acknowledgements}

None.

\section{Conflict of interest}

The author declares no conflict of interest.

\section{References}

1. Merskey H, Bogduk N. Classification of chronic pain. Descriptions of Chronic Pain Syndromes and Definitions of Pain Terms. IASP Press; 1994.

2. Salibi A, Searle AE, Lindau TR. Complex Regional Pain Syndrome: A systemic review of the literature. The past, present and future management. J Pain Relief. 2014;3:128.

3. Beumer A, Lindau TR. Grip strength ratio: a grip strength measurement that correlates well with DASH score in different hand/ wrist conditions. BMC Musculoskelet Disord. 2014;15(1):336.

4. Weiss DS, Marmar CR. The Impact of Event Scale-Revised. In J Wilson, et al. editors. Assessing psychological trauma and PTSD. New York: Guilford; 1996. p. 399-411.

5. Rosén B, Lundborg G. Training with a mirror in rehabilitation of the hand. Scandinavian j plast reconstr surg hand surg. 2005;39(2):104108 .

6. Moseley GL. Graded motor imagery is effective for long-standing complex regional pain syndrome: a randomised controlled trial. Pain. 2004;108(1-2):192-198.

7. Aktan ZA, Ozturk L, Calli H. An anatomical study of the first compartment of the wrist. Kaibougaku Zasshi. 1998;73(1):49-54.

8. Ramachandran VS, Hirstein W. The perception of phantom limbs. The DO Hebb lecture. Brain. 1998;121(9):1603-1630. 